Research Paper

\title{
The association between infection incidence and autoimmune diseases in breast cancer patients after anti-cancer treatment
}

\author{
Chien-Chih Chen ${ }^{1,2}$, Wei-Li Ho ${ }^{3}$, Hsin-Hua Chen1,4,5,6,7,8,9, Meei-Ling Sheu1,10, Chiann-Yi Hsu11, Jun-Peng \\ Chen ${ }^{11 \bowtie}$ \\ 1. Ph.D. Program in Translational Medicine, National Chung-Hsing University, Taichung, Taiwan \\ 2. Department of Radiation Oncology, Taichung Veterans General Hospital, Taichung, Taiwan \\ 3. Division of Allergy, Immunology and Rheumatology, Chiayi Branch, Taichung Veterans General Hospital \\ 4. Department of Medical Research, Taichung Veterans General Hospital, Taichung, Taiwan \\ 5. School of Medicine, National Yang-Ming University, Taipei, Taiwan \\ 6. Institute of Biomedical Science and Rong Hsing Research Center for Translational Medicine, Chung-Hsing University, Taichung, Taiwan \\ 7. School of Medicine, Chung-Shan Medical University, Taichung, Taiwan. \\ 8. Institute of Public Health and Community Medicine Research Center, National Yang-Ming University, Taipei, Taiwan \\ 9. Department of Industrial Engineering and Enterprise Information, Tunghai University, Taichung, Taiwan \\ 10. Graduate Institute of Biomedical Sciences, National Chung Hsing University, Taichung, Taiwan \\ 11. Biostatistics Task Force of Taichung Veterans General Hospital, Taichung, Taiwan
}

$\square$ Corresponding author: Hsin-Hua Chen, Division of Allergy, Immunology, and Rheumatology, Department of Internal Medicine, Taichung Veterans General Hospital, Taichung, Taiwan, No.1650, Sect. 4, Taiwan Boulevard, Taichung 40705, Taiwan, R.O.C. Tel: 886-4-23592525; Fax: 886-4-23741316; E-mail: shc5555@vghtc.gov.tw

(c) Ivyspring International Publisher. This is an open access article distributed under the terms of the Creative Commons Attribution (CC BY-NC) license (https://creativecommons.org/licenses/by-nc/4.0/). See http://ivyspring.com/terms for full terms and conditions.

Received: 2018.06.19; Accepted: 2018.12.08; Published: 2019.01.29

\begin{abstract}
Purpose: To evaluate the infection incidence in breast cancer patients whether they have a major autoimmune disease or not.

Methods: This retrospective cohort study compared the infection incidence of 174 breast cancer patients with an autoimmune disease, including Sjogren's Syndrome (SS), Rheumatoid Arthritis (RA), and Systemic Lupus Erythematosus (SLE), along with 4429 patients without an autoimmune disease, for the period 2000 to 2016. Six-hundred and ninety six, age-, stage-, and diagnosis era-matched patients without any autoimmune disease were analyzed to eliminate the effects of these confounding factors may have on the results.

Results: After adjusting for age, stage and diagnosis era, breast cancer patients with an autoimmune disease had a higher Infection Incidence Ratio (IRR: 2.62) than the patients without any autoimmune disease. In the univariate analysis, patients who had an autoimmune disease $(p<0.001)$, underwent chemotherapy $(p<0.001)$, radiotherapy $(p=0.004)$, and monoclonal antibody therapy $(p<0.001)$ had a higher infection rate. In the multivariate analysis, autoimmune disease was shown to be an independent factor for infection incidence.

Conclusion: Autoimmune disease was a potential predictor of infection incidence in breast cancer patients post-treatment after adjusting for clinical confounding factors.
\end{abstract}

Key words: breast cancer, infection, autoimmune disease

\section{Introduction}

Chronic inflammation plays an important shared role in the pathogenesis of both autoimmune diseases and cancer [1]. An autoimmune disease is a condition in which an abnormal immune system attacks normal tissue and leads to the chronic inflammation of multiple organs. Long-term immunosuppressive therapy for autoimmune disease not only affects the cancer treatment response, but also increases the infection rate [2-4].

The incidence rate of autoimmune diseases is approximately $8 \%$, with $78 \%$ of autoimmune disease patients being female [5]. Breast cancer is one of most common malignancies worldwide among women. To our knowledge, there are currently few data on breast 
cancer and autoimmune disease in the literature [6-10]. The association of autoimmune disease with infection incidence and clinical outcomes of breast cancer remains unclear. It has been hypothesized that autoimmune disease and immunosuppressive therapy may affect the immune response, thereby leading to increase risk of infection. A previous study [11] showed that infection episodes affected the overall survival of cancer patients. The purpose of this retrospective cohort study was to investigate the infection incidence and clinical outcomes in breast cancer patients with or without major autoimmune diseases, including Sjogren's Syndrome (SS), Rheumatoid Arthritis (RA), and Systemic Lupus Erythematosus (SLE). In order to adjust for clinical confounding factors, we compared the outcomes of the breast cancer patients with autoimmune disease with stage-, age- and diagnosis era- matched patients without autoimmune disease.

\section{Materials and Methods}

\section{Patients}

This retrospective cohort study reviewed a database of cancer patients who had been newly diagnosed with female breast cancer from January 2000 to December 2016 at Taichung Veterans General Hospital. The inclusion criteria for breast cancer patients with autoimmune disease were: (1) who had undergone a complete pretreatment staging workup, and (2) had pathologically confirmed breast cancer. The major autoimmune diseases included SS, RA, and SLE. The clinical outcomes and treatments, including surgery, radiotherapy, chemotherapy, hormone therapy, and monoclonal antibody therapy, were collected from our database. Other confounding factors, including diabetes mellitus (DM), hypertension (HTN), white blood count (WBC), liver function, and renal function, were also recorded and analyzed. The lab data, including WBC, liver function, and renal function, were collected within one month from the date of diagnosis and during the course of chemotherapy, radiotherapy, hormone therapy, or monoclonal antibody therapy. The abnormal lab data grading was performed according to Common Terminology Criteria for Adverse Events (CTCAE) 3.0. The hospitalized infection diagnosis was done according to international classification of diseases (ICD) code, and the infection diagnosis included infection of respiratory tract, urinary tract, skin and soft tissue, gastrointestinal tract, liver, biliary tract, cardiovascular system, bacteremia and septicemia, along with other opportunistic infections.

The inclusion criteria for breast cancer patients without autoimmune disease were: (1) pathologically confirmed breast cancer, and (2) no diagnosis of any autoimmune disease diagnosis, including SLE, RA, SS, Ankylosing Spondylitis, Psoriasis, Psoriatic Arthritis, Anti-phospholipid Antibody Syndrome, Polymyositis, Dermatomyositis, Systemic Sclerosis, Mixed Connective Tissue Disease, Multiple Sclerosis, Palindromic Rheumatism, Neuromyelitis Optica, Myasthenia Gravis, Hashimoto's disease, Immune Thrombocytopenia, Autoimmune Hemolytic Anemia, Juvenile Idiopathic Arthritis, adult onset Still's disease, Crohn's disease, Ulcerative Colitis, Wegener's granulomatosis, and Iritis from January 2000 to December 2016 at Taichung Veterans General Hospital.

There were 4429 breast cancer patients without an autoimmune disease and 174 breast cancer patients with an autoimmune disease. Propensity score matching method was used to adjust for confounding factors. After adjusting for stage, age and diagnosis era, there were 696 matched patients who were selected for analysis.

The study was approved by the Institutional Review Board of Taichung Veterans General Hospital.

\section{Statistical analysis}

The endpoints were infection incidence and overall survival (OS). The infection incidence was calculated from the date of cancer diagnosis to the date of any infection diagnosis reported in the medical records. Some patients may have had several infection events at different times, so we also calculated accumulation infection incidence (person-years). The OS was calculated from the date of cancer diagnosis to the date of death from any cause, or the date of last follow-up. Survival status was defined according to whether a patient was alive or dead at the date of the last follow-up. Chi-square test was used to compare categorical variables and the Mann-Whitney $U$ test was used to analyze the continuous variables. Survival times were estimated using the Kaplan-Meier method, while the Log-rank test was used for comparisons between the groups. Factors which displayed a tendency to affect outcomes in the univariate analysis were incorporated in the multivariate analysis. A Cox proportional hazard model was used for multivariate analysis. The statistical analyses were performed using SPSS software, version 22.0. A $p$ value less than 0.05 was considered statistically significant.

\section{Results}

Table 1 shows the accumulation infection incidence of all breast cancer patients with or without autoimmune disease. After adjusting for age, stage and diagnosis era, the breast cancer patients with 
autoimmune disease displayed a higher infection incidence ratio (IRR: 2.62) than patients without autoimmune disease (Figure 1, $\mathrm{p}<0.001$ ).

Table 2 summarizes the matched patients' characteristics. The median age of autoimmune disease group was 52 years old, ranging from 45 to 61 years old. The ratio of matched breast cancer patients with or without autoimmune disease was 1:4. The most common autoimmune disease in breast cancer patients was SS (66 patients, 37.9\%). The second and third most common autoimmune diseases were RA (64 patients, 36.8\%) and SLE (44 patients, 25.3\%). Patients with autoimmune disease experienced a higher infection incidence $(22.4 \%)$ than patients without autoimmune disease $(7.9 \%)$. There were more patients with DM, HTN, and abnormal WBC in the autoimmune disease group.

Table 3 summarizes the univariate analysis of infection incidence. In the univariate analysis, for patients with an autoimmune disease $(p<0.001)$, chemotherapy $(p<0.001)$, hormone therapy $(p=0.016)$, radiotherapy $(p=0.004)$, and monoclonal antibody therapy $(p<0.001)$ resulted in a higher infection incidence. Table 4 summarizes the univariate analysis of OS. The 5-year overall survival rates were $92.2 \%$ and $89.2 \%$ in breast cancer patients with and without autoimmune disease, respectively $(p=0.366$, Figure 2$)$. Patients with advanced stage cancer exhibited poor OS $(p<0.001)$, however autoimmune disease did not affect OS $(p=0.533)$ on univariate analysis. Patients with an infection diagnosis had poor OS $(\mathrm{p}<0.001)$.

Factors, including autoimmune disease and treatment, which had a tendency to affect the infection incidence in the univariate analysis, were incorporated into the multivariate analysis. Table 5 summarizes the multivariate analysis of infection incidence according to different autoimmune diseases. In the multivariate analysis, autoimmune diseases, including SS (Hazard Ratio [HR] $=2.34 ; 95 \%$ Confidence Interval $[\mathrm{CI}]=1.24-4.43 ; \mathrm{p}=0.009)$, RA $(\mathrm{HR}=2.94 ; 95 \% \quad \mathrm{CI}=1.66-5.20 ; \mathrm{p}<0.001)$, and SLE $(\mathrm{HR}=2.87 ; \quad 95 \% \quad \mathrm{CI}=1.45-5.68 ; \quad \mathrm{p}=0.002)$, were independent predictors of infection incidence.

Table 1. Incidence rate of infection

\begin{tabular}{|c|c|c|c|c|c|c|c|c|c|c|c|}
\hline & \multirow[t]{2}{*}{ Total } & \multirow[t]{2}{*}{ Event $(\%)$} & \multirow[t]{2}{*}{ Total person-years } & \multirow[t]{2}{*}{ Incidence Rate (/103 years) } & \multicolumn{2}{|c|}{ Crude } & \multicolumn{2}{|l|}{ IRR $^{a}$} & \multicolumn{2}{|c|}{ IRR $\mathrm{b}$} & IRRc \\
\hline & & & & & IRR & $95 \% \mathrm{CI}$ & IRR & $95 \% \mathrm{CI}$ & IRR & $95 \% \mathrm{CI}$ & IRR $95 \% \mathrm{CI}$ \\
\hline non-autoimmune disease & 4429 & $450(10.2 \%)$ & 19197.4 & 23.4 & 1 & & 1 & & 1 & & 1 \\
\hline Autoimmune disease & 174 & $39(22.4 \%)$ & 684.5 & 57.0 & 2.43 & $(1.75-3.37)$ & 2.35 & $(1.69-3.26)$ & 2.72 & $(1.96-3.78)$ & $2.62(1.88-3.63)$ \\
\hline
\end{tabular}

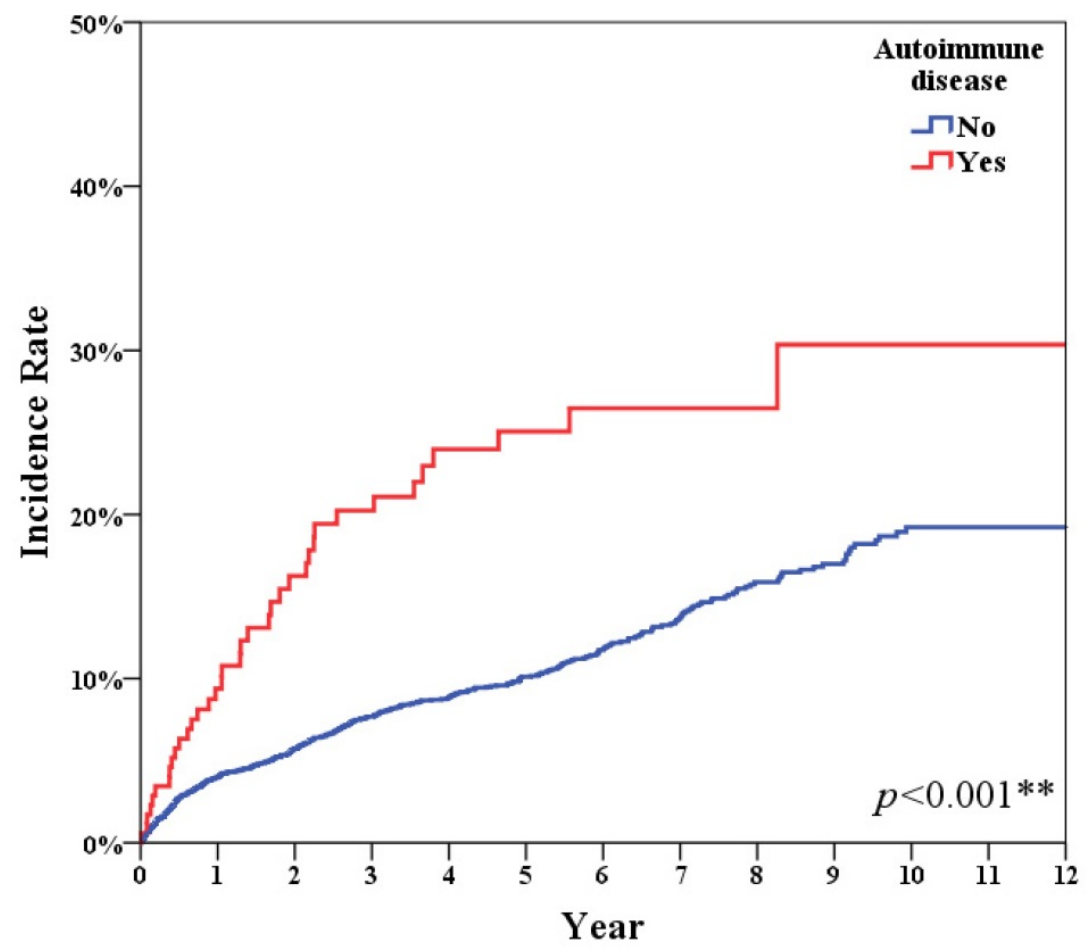

Figure 1. Autoimmune disease had significant higher infection incidence $(p<0.001)$. 
Table 2. Comparison of clinical characteristics of breast cancer patients with and without autoimmune disease

\begin{tabular}{|c|c|c|c|c|c|}
\hline \multirow[b]{3}{*}{ Infection diagnosis } & \multicolumn{4}{|c|}{ Autoimmune disease } & \multirow{3}{*}{$\begin{array}{l}p \text { value } \\
<0.001^{* *}\end{array}$} \\
\hline & \multicolumn{2}{|c|}{ No $(n=696)(\%)$} & \multicolumn{2}{|c|}{ Yes $(n=174)(\%)$} & \\
\hline & 55 & $(7.9)$ & 39 & $(22.4)$ & \\
\hline Age, year\& & & & & & 0.635 \\
\hline Median & 52 & & 52 & & \\
\hline IQR range & $45-60$ & & $45-61$ & & \\
\hline Stage (II-IV) & 296 & $(42.5)$ & 74 & $(42.5)$ & 1.000 \\
\hline Death & 65 & $(9.3)$ & 13 & $(7.5)$ & 0.533 \\
\hline Triple-negative breast cancer & 36 & $(5.2)$ & 7 & $(4.0)$ & 0.667 \\
\hline \multicolumn{6}{|l|}{ Treatment } \\
\hline Surgery & 673 & $(96.7)$ & 164 & $(94.3)$ & 0.198 \\
\hline Chemotherapy & 368 & $(52.9)$ & 106 & $(60.9)$ & 0.069 \\
\hline Hormone therapy & 480 & $(69.0)$ & 125 & $(71.8)$ & 0.519 \\
\hline Radiation therapy & 296 & $(42.5)$ & 79 & $(45.4)$ & 0.549 \\
\hline Target & 87 & $(12.5)$ & 24 & $(13.8)$ & 0.741 \\
\hline $\mathrm{DM}$ & 16 & $(2.3)$ & 16 & $(9.2)$ & $<0.001^{* *}$ \\
\hline Hypertension & 36 & $(5.2)$ & 35 & $(20.1)$ & $<0.001^{* *}$ \\
\hline $\mathrm{Hb}$ & & & & & 0.317 \\
\hline Normal & 479 & $(79.8)$ & 112 & $(75.7)$ & \\
\hline Abnormal & 121 & $(20.2)$ & 36 & $(24.3)$ & \\
\hline WBC & & & & & $<0.001^{* *}$ \\
\hline Normal & 584 & $(97.3)$ & 134 & $(90.5)$ & \\
\hline Abnormal & 16 & $(2.7)$ & 14 & $(9.5)$ & \\
\hline Renal function (Creatinine) ${ }^{\mathrm{f}}$ & & & & & 0.299 \\
\hline Normal & 590 & $(98.5)$ & 143 & $(97.3)$ & \\
\hline Abnormal & 9 & (1.5) & 4 & $(2.7)$ & \\
\hline Liver function (GOT/GPT) & & & & & 0.519 \\
\hline Normal & 555 & $(92.5)$ & 133 & $(90.5)$ & \\
\hline Abnormal & 45 & (7.5) & 14 & $(9.5)$ & \\
\hline \multicolumn{6}{|l|}{ Autoantibodies } \\
\hline \multicolumn{6}{|l|}{ RF-IgM } \\
\hline Positive & & & 29 & $(16.7)$ & \\
\hline Negative & & & 82 & $(47.1)$ & \\
\hline Not available & & & 63 & $(36.2)$ & \\
\hline \multicolumn{6}{|l|}{ ANA } \\
\hline Positive & & & 56 & $(32.2)$ & \\
\hline Negative & & & 70 & $(40.2)$ & \\
\hline Not available & & & 48 & $(27.6)$ & \\
\hline \multicolumn{6}{|l|}{ SSA } \\
\hline Positive & & & 69 & (39.7) & \\
\hline Negative & & & 9 & (5.1) & \\
\hline Not available & & & 96 & $(55.2)$ & \\
\hline \multicolumn{6}{|l|}{ SSB } \\
\hline Positive & & & 50 & $(28.7)$ & \\
\hline Negative & & & 28 & $(16.1)$ & \\
\hline Not available & & & 96 & $(55.2)$ & \\
\hline
\end{tabular}

\section{Discussion}

This retrospective cohort study is the first to analyze the association between comorbid autoimmune diseases with infection requiring hospitalization and OS based on 174 breast cancer patients with major autoimmune diseases and 696 age-, stage-, and diagnosis era-matched breast cancer patients without a major autoimmune disease. We found that the presence of a major autoimmune disease was an independent factor for infection incidence, after adjusting for clinical factors. The ability to identify patients with high risk of infection may be of value in clinical practice as physicians can apply appropriate medications and surveys.
It has been hypothesized that when autoimmune disease patients are regularly followed up at a hospital, more tumors can be detected at an early stage [12]. In our study, $40.4 \%$ of 4429 breast cancer patients without autoimmune disease had stage I breast cancer, while $57.5 \%$ of the 174 breast cancer patients with autoimmune disease had stage I breast cancer. In our study, advanced stage patients had poorer OS in the univariate analysis $(<0.001)$. After adjusting for stage factors, there was no significant difference in OS between breast cancer patients with or without autoimmune disease.

Table 3. Comparison of clinical characteristics between patients with and without infection diagnosis.

\begin{tabular}{|c|c|c|c|c|}
\hline & \multicolumn{3}{|c|}{ Infection diagnosis } & \multirow[t]{2}{*}{$p$ value } \\
\hline & \multicolumn{2}{|c|}{ No $(n=776)(\%)$} & Yes $(n=94)(\%)$ & \\
\hline Autoimmune disease & 135 & $(17.4)$ & $39(41.5)$ & $<0.001^{* *}$ \\
\hline Age, year\& & 52 & & 54 & 0.437 \\
\hline \multicolumn{5}{|l|}{ Median } \\
\hline \multicolumn{5}{|l|}{$\mathrm{IQR}$ range } \\
\hline Stage (II-IV) & 322 & $(41.5)$ & $48(51.1)$ & 0.097 \\
\hline Triple-negative breast cancer $\mathrm{f}$ & 41 & $(5.3)$ & $2(2.1)$ & 0.280 \\
\hline \multicolumn{5}{|l|}{ Treatment } \\
\hline Surgery $f$ & 748 & $(96.4)$ & $89(94.7)$ & 0.390 \\
\hline Chemotherapy & 403 & $(51.9)$ & $71(75.5)$ & $<0.001^{\text {** }}$ \\
\hline Hormone therapy & 529 & $(68.2)$ & $76(80.9)$ & $0.016^{*}$ \\
\hline Radiation therapy & 321 & $(41.4)$ & $54(57.4)$ & $0.004^{* *}$ \\
\hline Monoclonal antibody & 79 & $(10.2)$ & $32(34.0)$ & $<0.001^{* *}$ \\
\hline $\mathrm{DM}^{\mathrm{f}}$ & 26 & (3.4) & $6 \quad(6.4)$ & 0.145 \\
\hline Hypertension & 60 & $(7.7)$ & $11(11.7)$ & 0.259 \\
\hline $\mathrm{Hb}$ & & & & 0.051 \\
\hline Normal & 535 & $(80.1)$ & $56(70.0)$ & \\
\hline Abnormal & 133 & (19.9) & $24(30.0)$ & \\
\hline$W B C^{f}$ & & & & 0.358 \\
\hline Normal & 643 & $(96.3)$ & $75(93.8)$ & \\
\hline Abnormal & 25 & (3.7) & $5 \quad(6.3)$ & \\
\hline Renal function (Creatinine) $\mathrm{f}$ & & & & 0.641 \\
\hline Normal & 655 & $(98.3)$ & $78(97.5)$ & \\
\hline Abnormal & 11 & $(1.7)$ & $2(2.5)$ & \\
\hline Liver function (GOT/GPT) & & & & 0.023 \\
\hline Normal & 620 & $(93.0)$ & $68(85.0)$ & \\
\hline Abnormal & 47 & $(7.0)$ & $12(15.0)$ & \\
\hline \multicolumn{5}{|l|}{ Autoantibodies } \\
\hline RF-IgM & & & & 0.005 \\
\hline Positive & & & $5 \quad(5.3)$ & \\
\hline Negative & & & $19(20.2)$ & \\
\hline Not available & & & $70(74.5)$ & \\
\hline ANA & & & & $<0.001$ \\
\hline Positive & & & $13(13.8)$ & \\
\hline Negative & & & $25(26.6)$ & \\
\hline Not available & & & $56(59.6)$ & \\
\hline SSA & & & & $<0.001$ \\
\hline Positive & & & $19(20.2)$ & \\
\hline Negative & & & 1 (1.1) & \\
\hline Not available & & & $74(78.7)$ & \\
\hline SSB & & & & $<0.001$ \\
\hline Positive & & & $14(14.9)$ & \\
\hline Negative & & & $6 \quad(6.4)$ & \\
\hline Not available & & & $74(78.7)$ & \\
\hline
\end{tabular}


Table 4. Comparison of clinical characteristic between alive patients and those who died during the follow-up period

\begin{tabular}{|c|c|c|c|c|c|}
\hline \multirow[b]{3}{*}{ Autoimmune disease } & \multicolumn{4}{|c|}{ Survival status } & \multirow{3}{*}{$\begin{array}{l}p \text { value } \\
0.533\end{array}$} \\
\hline & \multicolumn{2}{|c|}{ Alive (n=792) (\%) } & \multicolumn{2}{|c|}{ Death $(\mathrm{n}=78)(\%)$} & \\
\hline & 161 & $(20.3)$ & 13 & $(16.7)$ & \\
\hline Infection diagnosis & 71 & $(9.0)$ & 23 & $(29.5)$ & $<0.001^{* * *}$ \\
\hline Age, year\& & & & & & $0.008^{* *}$ \\
\hline Median & 52 & & 58 & & \\
\hline IQR range & $45-60$ & & $45-68$ & & \\
\hline Stage(II-IV) & 305 & $(38.5)$ & 65 & $(83.3)$ & $<0.001^{\text {** }}$ \\
\hline Triple-negative breast cancer & 38 & $(4.8)$ & 5 & $(6.4)$ & 0.724 \\
\hline \multicolumn{6}{|l|}{ Treatment } \\
\hline Surgery ${ }^{f}$ & 767 & $(96.8)$ & 70 & $(89.7)$ & $0.006^{* *}$ \\
\hline Chemotherapy & 413 & (52.1) & 61 & $(78.2)$ & $<0.001^{* * *}$ \\
\hline Hormone therapy & 548 & $(69.2)$ & 57 & $(73.1)$ & 0.560 \\
\hline Radiation therapy & 340 & $(42.9)$ & 35 & $(44.9)$ & 0.833 \\
\hline Target ${ }^{\mathrm{f}}$ & 92 & (11.6) & 19 & $(24.4)$ & $0.002^{\star *}$ \\
\hline $\mathrm{DM}^{\mathrm{f}}$ & 28 & (3.5) & 4 & $(5.1)$ & 0.521 \\
\hline Hypertension & 65 & (8.2) & 6 & $(7.7)$ & 1.000 \\
\hline $\mathrm{Hb}$ & & & & & 0.126 \\
\hline Normal & 554 & $(79.7)$ & 37 & $(69.8)$ & \\
\hline Abnormal & 141 & (20.3) & 16 & $(30.2)$ & \\
\hline WBC ${ }^{f}$ & & & & & 1.000 \\
\hline Normal & 667 & $(96.0)$ & 51 & $(96.2)$ & \\
\hline Abnormal & 28 & $(4.0)$ & 2 & $(3.8)$ & \\
\hline Renal function (Creatinine) ${ }^{\mathrm{f}}$ & & & & & 1.000 \\
\hline Normal & 681 & $(98.3)$ & 52 & $(98.1)$ & \\
\hline Abnormal & 12 & $(1.7)$ & 1 & $(1.9)^{\prime}$ & \\
\hline Liver function $(G O T / G P T)^{\mathrm{f}}$ & & & & & 0.599 \\
\hline Normal & 640 & $(92.2)$ & 48 & $(90.6)$ & \\
\hline Abnormal & 54 & $(7.8)$ & 5 & $(9.4)$ & \\
\hline \multicolumn{6}{|l|}{ Autoantibodies } \\
\hline RF-IgM & & & & & 0.017 \\
\hline Positive & & & 0 & $(0)$ & \\
\hline Negative & & & 3 & (3.8) & \\
\hline Not available & & & 75 & $(96.2)$ & \\
\hline ANA & & & & & 0.005 \\
\hline Positive & & & 3 & (3.8) & \\
\hline
\end{tabular}

\begin{tabular}{|c|c|c|c|c|}
\hline \multirow{3}{*}{ Negative } & \multicolumn{3}{|l|}{ Survival status } & \multirow[t]{2}{*}{$p$ value } \\
\hline & Alive $(\mathrm{n}=792)(\%)$ & Dea & $(\mathrm{n}=78)(\%)$ & \\
\hline & & 3 & (3.8) & \\
\hline Not available & & 72 & (92.4) & \\
\hline SSA & & & & 0.176 \\
\hline Positive & & 3 & (3.8) & \\
\hline Negative & & 0 & (0) & \\
\hline Not available & & 75 & $(96.2)$ & \\
\hline SSB & & & & 0.132 \\
\hline Positive & & 3 & (3.8) & \\
\hline Negative & & 0 & $(0)$ & \\
\hline Not available & & 75 & $(96.2)$ & \\
\hline
\end{tabular}

Park et al. [7] analyzed 122 cancer patients with autoimmune disease and found that Dermatomyositis and Polymyositis (PM) increased mortality in breast cancer patients. In Park's study, only $22.5 \%$ of the patients had breast cancer, and the number of males was higher than that of females in the Dermatomyositis and PM groups. In our study, we limited the scope of the analysis to female breast cancer patients in order to eliminate the effects of gender. In addition, the main focus of the present study was the three most common autoimmune diseases found in breast cancer patients. Our study showed no significant difference in OS.

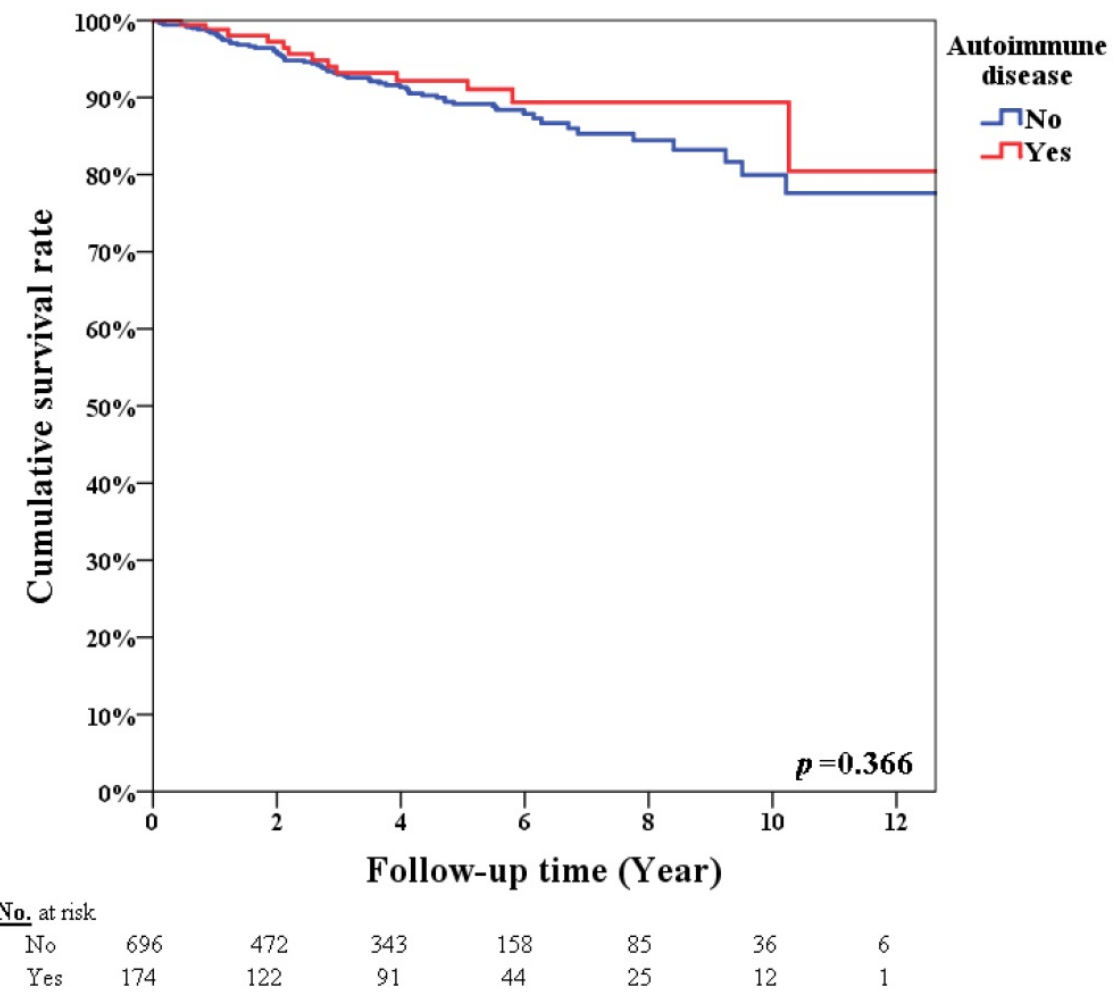

Figure 2. Survival rate according to patients with or without autoimmune disease $(p=0.366)$. 
Table 5. Multivariate analysis of infection incidence (matching: Age, stage and diagnosis era)

\begin{tabular}{|c|c|c|c|c|c|c|c|c|c|c|c|c|}
\hline & \multicolumn{3}{|c|}{ All autoimmune disease } & \multicolumn{3}{|l|}{ SS } & \multicolumn{3}{|l|}{ RA } & \multicolumn{3}{|l|}{ SLE } \\
\hline & HR & $95 \% \mathrm{CI}$ & $p$ value & $\mathrm{HR}$ & $95 \% \mathrm{CI}$ & $p$ value & HR & $95 \% \mathrm{CI}$ & $p$ value & HR & $95 \% \mathrm{CI}$ & $p$ value \\
\hline Autoimmune disease & 2.18 & $1.48-3.21$ & $<0.01^{* *}$ & 2.34 & $1.24-4.43$ & $0.009^{* *}$ & 2.94 & $1.66-5.20$ & $<0.001^{* *}$ & 2.87 & $1.45-5.68$ & $0.002^{* *}$ \\
\hline Treatment & & & & & & & & & & & & \\
\hline Surgery & 0.33 & $0.23-0.48$ & $<0.01^{* *}$ & 0.27 & $0.08-0.92$ & $0.036^{*}$ & 0.36 & $0.12-1.08$ & 0.069 & 0.18 & $0.06-0.54$ & $0.002^{* *}$ \\
\hline Chemotherapy & 1.83 & $1.34-2.51$ & $<0.01^{* *}$ & 1.48 & $0.80-2.77$ & 0.214 & 1.73 & $0.94-3.17$ & 0.076 & 1.92 & $1.01-3.65$ & $0.048^{*}$ \\
\hline Hormone therapy & 1.24 & $0.98-1.57$ & 0.07 & 1.93 & $1.05-3.52$ & $0.033^{*}$ & 1.95 & $1.07-3.54$ & $0.029^{*}$ & 1.72 & $0.94-3.12$ & 0.077 \\
\hline Radiation therapy & 1.10 & $0.88-1.38$ & 0.40 & 2.13 & $1.24-3.67$ & $0.006^{* *}$ & 1.24 & $0.74-2.08$ & 0.422 & 1.72 & $1.02-2.90$ & $0.043^{*}$ \\
\hline Target therapy & 2.12 & $1.68-2.67$ & $<0.01^{* *}$ & 4.54 & $2.58-7.97$ & $<0.001^{* *}$ & 2.47 & $1.42-4.30$ & $0.001^{* *}$ & 3.08 & $1.76-5.37$ & $<0.001^{* *}$ \\
\hline
\end{tabular}

Cox regression. ${ }^{*} p<0.05,{ }^{* *} p<0.01$.

Previous studies [13-16] have demonstrated that autoimmune disease patients have different types of immune dysregulation and that these defects lead to chronic inflammation, organ injury, and poor response to infection. To the best of our knowledge, there are few data in the literature on infection incidence and autoimmune disease in breast cancer patients. In our study, we found that autoimmune disease was an independent predictor of infection incidence in breast cancer patients.

This retrospective study had some limitations. First, stage, age, and diagnosis era all affected infection incidence. Second, different treatment options depended on the particular disease, patients' condition, physicians' decision, and diagnosis era, all of which may have affected the clinical results and the possibility of infection. Third, autoimmune disease likely affected the physician's treatment choice. It is difficult to analyze the effects of different cancer treatments on infection incidence. In order to eliminate these confounding factors, 696 stage-, age-, and diagnosis era-matched patients were selected for this study. All of the different treatments, including surgery, radiotherapy, chemotherapy, monoclonal antibody therapy, along with the various autoimmune diseases were incorporated into the multivariate analysis in order to clarify the effects of different cancer treatments and autoimmune disease on infection incidence in breast cancer patients. Fourth, autoimmune disease status may affect the clinical outcomes and autoantibody profiles are associated with the activity of cancers as well as those of autoimmune diseases. It is particularly challenging to identify which period during treatment and follow-up and which autoantibody has the most important effect on infection incidence and survival. The autoantibody profiles are presented in the tables and the incidence rate of infection and death are shown in the supplemental tables. However, autoantibody profiles were not included in the multivariable analyses because few or none of the patients with positive autoantibodies either died or had infection eipsodes. Further studies are still required to determine the association between autoimmune disease and infection incidence in breast cancer patients.

\section{Conclusion}

Autoimmune disease was a potential predictor of infection incidence after adjusting for clinical confounding factors.

\section{Supplementary Material}

Supplementary tables.

http://www.jcancer.org/v10p0829s1.pdf

\section{Acknowledgements}

This study was supported by Taichung Veterans General Hospital.

\section{Ethics approval and consent to participate}

The study was approved by the Institutional Review Board of Taichung Veterans General Hospital.

\section{Competing Interests}

The authors have declared that no competing interest exists.

\section{References}

1. Franks AL, Slansky JE. Multiple associations between a broad spectrum of autoimmune disease, chronic inflammatory disease and cancer. Anticancer Res. 2012; 32: 1119-1136.

2. Vajdic CM, van Leeuwen MT. Cancer incidence and risk factors after solid organ transplantation. Int J Cancer. 2009; 125: 1747-1754.

3. Turesson C, Matteson EL. Malignancy as a comorbidity in rheumatic diseases. Rheumatology. 2013; 52: 5-14.

4. Swann JB, Smyth MJ. Immune surveillance of tumors. J Clin Invest. 2007; 117: 1137-1146.

5. Fairweather D, Frisancho-Kiss S, Rose NR. Sex differences in autoimmune disease from a pathological perspective. The Am J of pathol. 2008; 173: 600-609.

6. Hsu CY, Lin MS, Su YJ, et al. Cumulative immunosuppressant exposure is associated with diversified cancer risk among 14832 patients with systemic lupus erythematosus: a nested care control study. Rheum 2017; 56: 620-628.

7. Park JK, Yang JA, Ahn EY, et al. Survival rates of cancer patients with and without rheumatic disease: a retrospective cohort analysis. BMC Cancer. 2016; 16: 381-389.

8. Cloutier BT, Clarke AE, Ramsey-Goldman R, et al. Breast cancer in systemic lupus erythematosus. Oncol. 2013; 85: 117-121.

9. Bernatsky S, Ramsey-Goldman R, Petri M, et al. Breast cancer in systemic lupus. Lupus. 2017; 26: 311-315.

10. Khaliq W, Qayyum R, Clough J, et al. Comparison of breast cancer risk in women with and without systemic lupus erythematosus in a medicare population. Breast Cancer Res. Treat. 2015; 151: 465-474.

11. Thai V, Lau F, Wolch G, et al. Impact of infections on the survival of hospitalized advanced cancer patients. J Pain Symptom Manage. 2012; 43: 549-557.

12. Hemminki K, Liu X, Ji J, et al. Effect of autoimmune disease on mortality and survival in subsequent digestive tract cancers. Ann Oncol. 2012; 23: 2179-2184.

13. Schett $G$, Elewaut D, Mclnnes IB, et al. How cytokine networks fuel inflammation: toward a cytokine-based disease taxonomy. Nat Med. 2013; 19: 822-824.

14. Ho YY, Lagares D, Tager AM, et al. Fibrosis-a lethal component of systemic sclerosis. Nat Rev Rheumatol. 2014; 10: 390-402. 
15. Sakkas LI, Chikanza IC, Platsoucas CD. Mechanisms of disease: the role of immune cells in the pathogenesis of systemic sclerosis. Nat Clin Pract Rheumatol. 2006; 2: 679-685.

16. Deng GM, Tsokos GC. Pathogenesis and targeted treatment of skin injury in SLE. Nat Rev Rheumatol. 2015; 11: 663-669. 